\title{
A Novel COVID-19 Diagnosis Support System Using the Stacking Approach and Transfer Learning Technique on Chest $X$-Ray Images
}

\author{
Soufiane Hamida ${ }^{D},{ }^{1}$ Oussama El Gannour ${ }^{D},{ }^{1}$ Bouchaib Cherradi $\left(\mathbb{D},{ }^{1,2}\right.$ \\ Abdelhadi Raihani $\left(\mathbb{D},{ }^{1}\right.$ Hicham Moujahid $\left(\mathbb{D},{ }^{1}\right.$ and Hassan Ouajji ${ }^{1}$ \\ ${ }^{1}$ SSDIA Laboratory, ENSET of Mohammedia, Hassan II University of Casablanca, BP 159, Mohammedia, Morocco \\ ${ }^{2}$ STIE Team, CRMEF Casablanca-Settat, Provincial Section of El Jadida, 24000 El Jadida, Morocco \\ Correspondence should be addressed to Bouchaib Cherradi; bouchaib.cherradi@gmail.com
}

Received 29 May 2021; Revised 26 August 2021; Accepted 7 October 2021; Published 5 November 2021

Academic Editor: Matteo Russo

Copyright (c) 2021 Soufiane Hamida et al. This is an open access article distributed under the Creative Commons Attribution License, which permits unrestricted use, distribution, and reproduction in any medium, provided the original work is properly cited.

\begin{abstract}
COVID-19 is an infectious disease-causing flu-like respiratory problem with various symptoms such as cough or fever, which in severe cases can cause pneumonia. The aim of this paper is to develop a rapid and accurate medical diagnosis support system to detect COVID-19 in chest X-ray images using a stacking approach combining transfer learning techniques and KNN algorithm for selection of the best model. In deep learning, we have multiple approaches for building a classification system for analyzing radiographic images. In this work, we used the transfer learning technique. This approach makes it possible to store and use the knowledge acquired from a pretrained convolutional neural network to solve a new problem. To ensure the robustness of the proposed system for diagnosing patients with COVID-19 using X-ray images, we used a machine learning method called the stacking approach to combine the performances of the many transfer learning-based models. The generated model was trained on a dataset containing four classes, namely, COVID-19, tuberculosis, viral pneumonia, and normal cases. The dataset used was collected from a six-source dataset of X-ray images. To evaluate the performance of the proposed system, we used different common evaluation measures. Our proposed system achieves an extremely good accuracy of $99.23 \%$ exceeding many previous related studies.
\end{abstract}

\section{Introduction}

The SARS-CoV-2 has caused the borders of many countries to be closed and millions of citizens to be confined to their homes due to infection rates; there have been more than 147 million confirmed cases worldwide at this time (April 25, 2021). This virus originated in China in December 2019. At that time, China succeeded in containing the virus for almost three months after the start of the crisis. As of March 2020, Europe was the focus for the germination of the virus, where it infected more than 445000 people [1,2]. Italy became the country which recorded the highest death toll, followed by Spain, which overtook the Asian countries in death toll. This number is continuously increasing. Clinical features of infected COVID-19 cases include fever, respiratory symptoms, cough, dyspnea, and viral pneumonia [3]. The COVID-19 test is based on taking samples from the respiratory tract [4]. A high number of tests may prove to be the key tool to stop the virus spread in some countries. However, it is important to find and develop alternative methods to perform these tests quickly and efficiently.

Artificial Intelligence (AI) techniques have been widely used in many applications such as handwriting recognition [5], rumors or fake news detection in social media [6,7], medical diagnosis support systems (MDSS) [8,9], prediction of patients with heart disease [10-14], and MRI image segmentation [15-21]. Particularly in the medical field, these techniques have been proved invaluable in predicting 
positive cases of multiple diseases [14,22]. In the context of the COVID-19 pandemic spread, AI techniques have gained particular interest in terms of predicting positive cases based on different medical data. In addition, many computer-aided diagnosis (CAD) systems based on AI techniques for COVID-19 prediction using principally chest X-ray as input data have been proposed [23-27].

The main contribution of our paper is to propose and implement a novel architecture of an automatic detection system as an alternative diagnostic option to prevent the coronavirus spread [28]. This study is based on the combination of six different sources of X-ray image datasets. From these datasets, we generate a new radiographic image dataset containing four classes, namely, normal, COVID-19, tuberculosis, and pneumonia. The application of image processing allows image standardization and improves model learning.

Furthermore, we aim to improve the prediction accuracy of COVID-19. Thus, the proposed system combines five transfer learning (TL) algorithms, namely, ResNet152V2, ResNet101V2, MobileNetV2, VGG16, and VGG19. These TL-based models automatically extract the radiographic images' features. Then, we implemented the stacking technique and the KNN algorithm to combine the performances of the five generated classifiers models and make the final prediction. Indeed, this method can help choose the best model to detect patients infected with COVID-19. The main contribution of this paper consists of the development of an accurate medical diagnosis support system to detect COVID-19 in chest X-ray images using a stacking approach combining transfer learning techniques and KNN algorithm for the choice of the best model. To reach this objective, we trained and tested the proposed system on a consistent dataset of normal, viral pneumonia, tuberculosis, and COVID-19 cases. Then, the best generated model was compared with some models from the literature. Finally, the obtained results in terms of common metrics were compared to the state-of-the-art models.

The rest of this paper is organized as follows: Section 2 presents some relevant related work. Section 3 describes the materials and methods used, the dataset used, and TL algorithms and methodology followed. Experimental results and discussion are given in Section 4. Finally, we conclude our work in Section 5 with some future perspectives.

\section{Review of Some Related Works}

In the last year, researchers have developed and published many works with the goal of combating the SARS-CoV-2 global pandemic [29-32]. In the field of diagnosis, many studies used Artificial Intelligence techniques to process X-ray images and detect the effect in terms of percentage of the virus in a patient's lungs. Deep learning approaches are the most frequently used in image classification to achieve better results than those using traditional ML approaches [33]. In this section, we limit our investigation to some research using TL technique-based models to diagnose COVID-19 [34].
In [35], the ResNet50 network-based deep transfer learning model achieved $93.01 \%$ accuracy for a binary classification of cases with and without COVID-19. This model uses CT type images taken from two open-source datasets.

Other research proposes a new architecture for the detection of cases infected by COVID-19 called CovXNet [36]. This architecture is based on a deep CNN. The authors used 5,856 chest X-ray images composed of four classes: COVID-19, normal, viral pneumonia, and bacterial pneumonia. The CovXNet model achieved an accuracy of $89.6 \%$ for 3 classes and $90.6 \%$ for 4 classes.

Another model called CoroNet presented in [37] proposes a deep convolutional network, based on the Xception architecture. This model was trained on radiographic images collected from two public databases. This model reached a classification accuracy of $99 \%$ for 2 classes, $95 \%$ for 3 classes, and $89.6 \%$ for 4 classes.

A comparative study published in [38] presented a system based on 10 transfer learning-based models, namely, AlexNet, VGG16, VGG19, SqueezeNet, GoogLeNet, MobileNetV2, ResNet18, ResNet50, ResNet101, and finally Xception. The models trained on a database containing two classes: COVID-19 and viral pneumonia. The authors concluded that ResNet101 is the best model for the reliable detection of COVID-19 with an accuracy of $99.51 \%$.

The proposed study in [39] uses various deep learning architectures such as VGG16, DenseNet121, Xception, NASNet, and EfficientNet, to develop a diagnosis support system of COVID-19. The dataset used contains three classes, and the highest accuracy obtained was $93.48 \%$ by EfficientNet.

In [40], the authors proposed a model based on AlexNet architecture for feature extraction and classification of the X-ray images. They used a strength Pareto evolutionary algorithm-II (SPEA-II) to select the best hyperparameters for this model. The proposed model reached an accuracy of 99.13\% with a multiclass database.

Another study published in [41] proposed a diagnostic system based on the majority voting method according to the results given by five classifiers: MobileNetV2, ResNet50V2, ResNet50V1, DenseNet201, and ResNet11. This system is based on X-ray image dataset with the three classes of COVID-19, viral pneumonia, and normal. The best accuracy obtained by this model was $99.31 \%$.

In [42], the authors proposed an automated diagnostic model of chest X-rays involving COVID-19. The proposed model uses the truncated DenseNet network based on TL, partial layer freezing, and feature fusion named FusedDenseNet-Tiny. The proposed model reached a maximal accuracy of $97.99 \%$ with only 1.2 million parameters.

In [43], the authors proposed a deep learning model based on pretrained models using majority voting. To build this model, open-source chest X-ray images of normal, pneumonia, and COVID-19 cases were used in this study. The proposed model achieved an accuracy of $99.26 \%$.

Other research published in [44] offered three pretrained models for building a diagnostic system: ResNet50V2, VGG16, and Inception V3. For this study, the dataset used 
was obtained from two publicly available data sources, containing three classes: COVID-19, normal, and pneumonia X-ray images. The best accuracy achieved by this model was $95.49 \%$.

In [45], The VGG16 network based on transfer learning model achieved an accuracy of $91.69 \%$ in a multiclassification of COVID-19-infected, normal, and pneumonia cases. The model proposed in this study is based on $\mathrm{X}$-ray images from a publicly available dataset.

In [46], three pretrained transfer learning models were proposed: VGG16, Inception V3, and lastly EfficientNetB0. COVID-19, normal, and viral pneumonia X-ray images were the three classes in the dataset used in this study, which was compiled from a variety of public sources. For VGG16, Inception V3, and EfficientNetB0, the accuracy of the proposed models was $87.84 \%, 91.32 \%$, and $92.93 \%$, respectively. Table 1 summarizes these works by citing the number of classes, the models used, and the best reached values in terms of evaluation metrics.

\section{Materials and Methods}

3.1. Global Overview on the Proposed COVID-19 Diagnosis System. In this paper, we proposed a diagnosis system based on stacking technique using various TL models for detecting patients infected with COVID-19. We selected five powerful TL models available in the Keras library. The dataset used was based on six-source databases containing chest X-ray images. From these datasets, we generated a new database with four classes, normal, COVID-19, pneumonia, and tuberculosis [47]. Furthermore, we split the dataset obtained into three parts, training set, validation set, and testing set. Then, we started by applying a series of preprocessing steps to the dataset images. All the chest X-ray images were normalized to the same dimension of $224 \times 224 \times 3$.

The training step involves two levels to generate the final model. The first training level is the Base-Models as the wellknown models in TL, namely, ResNet152V2, ResNet101V2, MobileNetV2, VGG16, and VGG19. These models take as input the training set with a default dimension of $224 \times 224 \times 3$. Moreover, we define and detail in Section 3.4 the parameters of each proposed TL-based model. After building the models, we used a validation set to avoid overfitting or underfitting problems. Then, we used the testing set to classify and predict classes. The output prediction obtained in the first training level would be used as input at the metalevel. In the second level of the training phase, we applied the stacking technique to combine the predictions made by each classifier. This technique used the KNN algorithm [48-50], to make the final prediction by contributing to the performance of Base-Models. Finally, we saved the generated model and evaluated the proposed model performance. Figure 1 describes the main stages of building a COVID-19 diagnosis system architecture.

3.2. Stacking Technique. Stacking is one of the most frequently used ensemble methods in ML. The overall idea of this technique is to build many models with completely different algorithmic program types to achieve a final prediction. This method uses another algorithm to learn how to combine predictions from various ML algorithms [51]. Therefore, the input for this final algorithm is the prediction outputs of these various base algorithms. The input of this model is an ensemble which includes $n$ classification models. Figure 2 shows the steps followed to apply the stacking technique.

3.3. Dataset Description. As mentioned before, this work relied on the exploration of six different sources of chest $\mathrm{X}$-ray image datasets. In Figure 3, we present some samples from these datasets.

A diversity of datasets allowed us to increase the size of the dataset used in this study. In addition, this will ensure an improvement in terms of detection performance. Table 2 summarizes all the datasets explored in order to generate a final dataset containing four classes: COVID-19, tuberculosis, viral pneumonia, and normal.

After preparing our dataset, we split it into three parts as follows: $80 \%$ for training, $10 \%$ for validation, and $10 \%$ for testing. The images used in this study did not have fixed dimensions as all came from various reliable sources. For this, we proceeded to resize and normalize all X-ray images to $1024 \times 1024$. Table 3 represents the distributions of the dataset images by class.

\subsection{Tuned Pretrained Models Based on TL Technique}

3.4.1. Tuned ResNet152V2-Based Model. The original version of ResNetV2 convolutional neural network (CNN) architecture contains two models, namely, ResNet152V2 and ResNet101V2. These models were developed by Microsoft Research Asia (https://www.microsoft.com/enus/research/lab/microsoft-research-asia/) based on the ResNetV1 (https://github.com/tensorflow/models/blob/ master/research/slim/nets/resnet_v1.py) model in 2016, with different optimizers for each layer to improve the accuracy. In this work, we used ResNet152V2 that reached an accuracy of $94.2 \%$ based on the ImageNet dataset. Accordingly, we added some convolutional, flatten, and dense layers after the original version. The architecture details of the proposed tuned version based on ResNet152V2 are presented in Table 4.

The architecture consisted of 70525188 total parameters, with 70381444 trainable parameters and 143744 nontrainable parameters.

3.4.2. Tuned ResNet101V2-Based Model. The second model from the ResNetV2 family is ResNet101V2. This CNN model was formed on the ImageNet dataset. In addition, it reached an accuracy of $93.8 \%$. Table 5 illustrates the architecture details of this tuned model.

The architecture consisted of 54820100 total parameters, with 54722436 trainable parameters and 97664 nontrainable parameters. 
TABLE 1: Summary of related work.

\begin{tabular}{|c|c|c|c|c|c|c|}
\hline Works & Number of classes & Models used & Accuracy & Precision & Sensitivity & Specificity \\
\hline$[35]$ & $\begin{array}{c}\text { COVID-19 } \\
\text { Non-COVID-19 }\end{array}$ & ResNet50 & $93.01 \%$ & $95.18 \%$ & $91.45 \%$ & $94.77 \%$ \\
\hline$[36]$ & $\begin{array}{c}\text { COVID-19 } \\
\text { Normal } \\
\text { Viral pneumonia } \\
\text { Bact pneumonia } \\
\text { COVID-19 } \\
\text { Viral pneumonia } \\
\text { Bact pneumonia }\end{array}$ & CovXNet & $90.2 \%$ & $90.8 \%$ & $89.9 \%$ & $87.6 \%$ \\
\hline$[37]$ & $\begin{array}{c}\text { COVID-19 } \\
\text { Normal } \\
\text { Viral pneumonia } \\
\text { Bact pneumonia } \\
\text { COVID-19 } \\
\text { Normal } \\
\text { Bact pneumonia } \\
\end{array}$ & CoroNet & $89.6 \%$ & $95 \%$ & $89.92 \%$ & $96.4 \%$ \\
\hline [38] & $\begin{array}{l}\text { COVID-19 } \\
\text { Viral pneumonia }\end{array}$ & $\begin{array}{c}\text { AlexNet } \\
\text { VGG16 } \\
\text { VGG19 } \\
\text { SqueezeNet } \\
\text { GoogLeNet } \\
\text { MobileNetV2 } \\
\text { ResNet18 } \\
\text { ResNet50 } \\
\text { ResNet101 } \\
\text { Xception }\end{array}$ & $\begin{array}{c}78.92 \% \\
83.33 \% \\
85.29 \% \\
82.84 \% \\
85.29 \% \\
92.16 \% \\
91.61 \\
94.12 \% \\
99.51 \% \\
99.02 \%\end{array}$ & $\begin{array}{l}\text { N/A } \\
\text { N/A } \\
\text { N/A } \\
\text { N/A } \\
\text { N/A } \\
\text { N/A } \\
\text { N/A } \\
\text { N/A } \\
\text { N/A } \\
\text { N/A }\end{array}$ & $\begin{array}{c}89.21 \% \\
80.39 \% \\
92.16 \% \\
78.43 \% \\
81.37 \% \\
97.06 \% \\
95.10 \% \\
90.20 \% \\
100 \% \\
98.04 \%\end{array}$ & $\begin{array}{c}68.63 \% \\
86.27 \% \\
78.43 \% \\
87.52 \% \\
90.20 \% \\
87.25 \% \\
88.23 \% \\
100 \% \\
99.02 \% \\
100 \%\end{array}$ \\
\hline$[39]$ & $\begin{array}{c}\text { Normal } \\
\text { COVID-19 } \\
\text { Other } \\
\end{array}$ & $\begin{array}{c}\text { VGG16 } \\
\text { DenseNet121 } \\
\text { Xception } \\
\text { NASNet } \\
\text { EfficientNet } \\
\end{array}$ & $\begin{array}{l}79.01 \% \\
89.96 \% \\
88.03 \% \\
85.03 \% \\
93.48 \% \\
\end{array}$ & $\begin{array}{l}\text { N/A } \\
\text { N/A } \\
\text { N/A } \\
\text { N/A } \\
\text { N/A }\end{array}$ & $\begin{array}{l}\text { N/A } \\
\text { N/A } \\
\text { N/A } \\
\text { N/A } \\
\text { N/A } \\
\end{array}$ & $\begin{array}{l}\text { N/A } \\
\text { N/A } \\
\text { N/A } \\
\text { N/A } \\
\text { N/A } \\
\end{array}$ \\
\hline$[40]$ & $\begin{array}{l}\text { COVID-19 } \\
\text { Healthy } \\
\text { Pneumonia } \\
\text { Tuberculosis }\end{array}$ & AlexNet & $99.13 \%$ & N/A & $99.47 \%$ & $99.15 \%$ \\
\hline$[41]$ & $\begin{array}{c}\text { COVID-19 } \\
\text { Normal } \\
\text { Viral pneumonia }\end{array}$ & Majority voting method & $99.31 \%$ & $100 \%$ & $100 \%$ & N/A \\
\hline$[42]$ & $\begin{array}{l}\text { COVID-19 } \\
\text { Normal } \\
\text { Pneumonia } \\
\end{array}$ & DenseNet & $97.99 \%$ & $98.38 \%$ & $98.15 \%$ & N/A \\
\hline [43] & $\begin{array}{c}\text { COVID-19 } \\
\text { Normal } \\
\text { Pneumonia } \\
\end{array}$ & Majority voting method & $99.26 \%$ & $97.87 \%$ & $100 \%$ & $98.89 \%$ \\
\hline$[44]$ & $\begin{array}{l}\text { COVID-19 } \\
\text { Normal } \\
\text { Pneumonia } \\
\end{array}$ & $\begin{array}{c}\text { ResNet50V2 } \\
\text { VGG16 } \\
\text { Inception V3 }\end{array}$ & $\begin{array}{l}95.49 \% \\
92.70 \% \\
92.97 \% \\
\end{array}$ & $\begin{array}{l}96.85 \% \\
97.50 \% \\
97.60 \% \\
\end{array}$ & $\begin{array}{l}99.19 \% \\
94.35 \% \\
98.39 \% \\
\end{array}$ & $\begin{array}{l}98.27 \% \\
98.69 \% \\
98.67 \% \\
\end{array}$ \\
\hline$[45]$ & $\begin{array}{c}\text { COVID-19 } \\
\text { Normal } \\
\text { Pneumonia } \\
\end{array}$ & VGG16 & $91.69 \%$ & $92.33 \%$ & $95.92 \%$ & $100 \%$ \\
\hline$[46]$ & $\begin{array}{c}\text { COVID-19 } \\
\text { Normal } \\
\text { Viral pneumonia }\end{array}$ & $\begin{array}{c}\text { VGG16 } \\
\text { Inception V3 } \\
\text { EfficientNetB0 }\end{array}$ & $\begin{array}{l}87.84 \% \\
91.32 \% \\
92.93 \%\end{array}$ & $\begin{array}{l}82.00 \% \\
87.54 \% \\
88.30 \%\end{array}$ & $\begin{array}{l}82.33 \% \\
89.00 \% \\
90.00 \%\end{array}$ & $\begin{array}{l}91.20 \% \\
94.00 \% \\
95.00 \%\end{array}$ \\
\hline
\end{tabular}




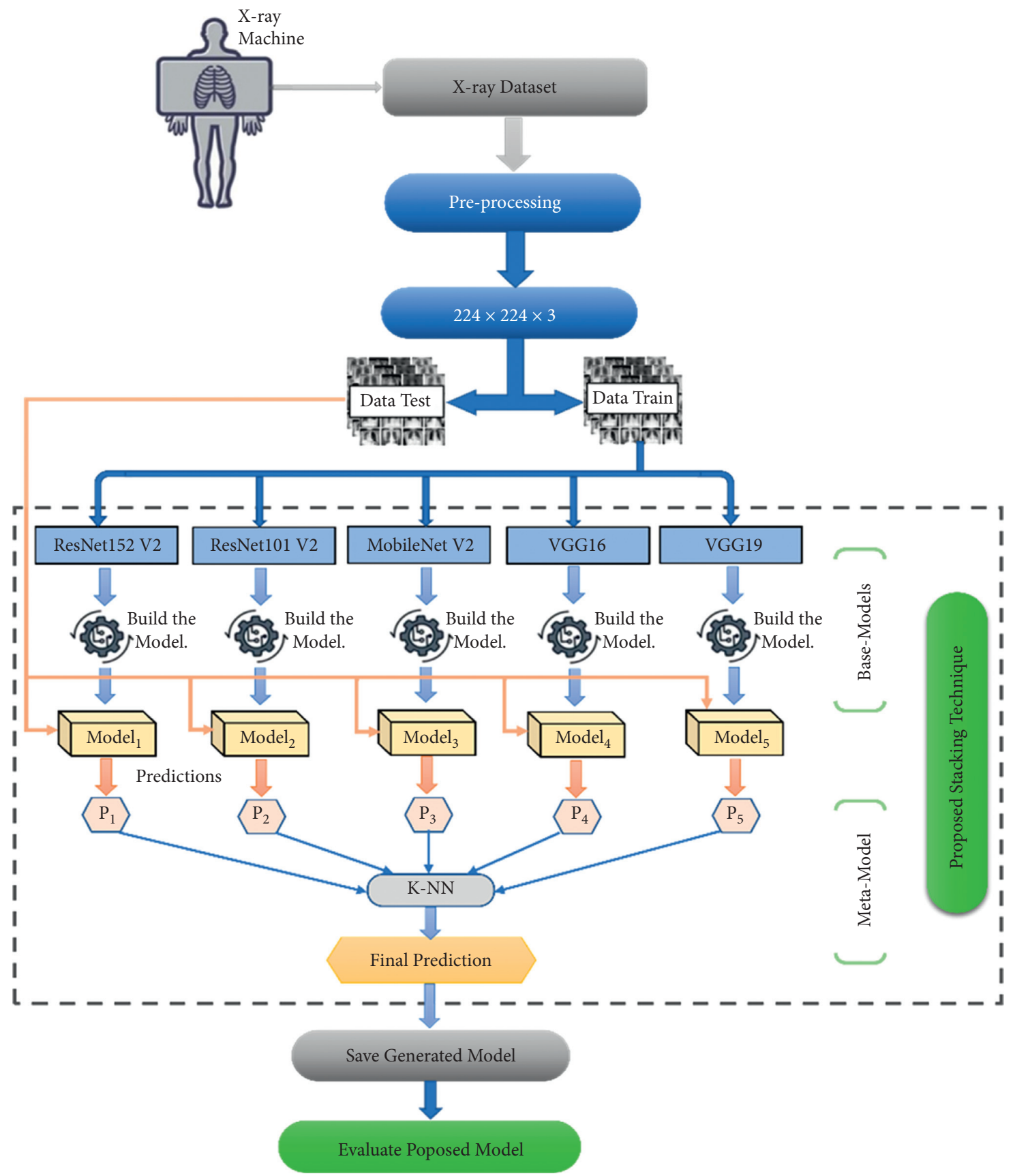

FIGURE 1: Flowchart representing the proposed diagnosis system architecture.

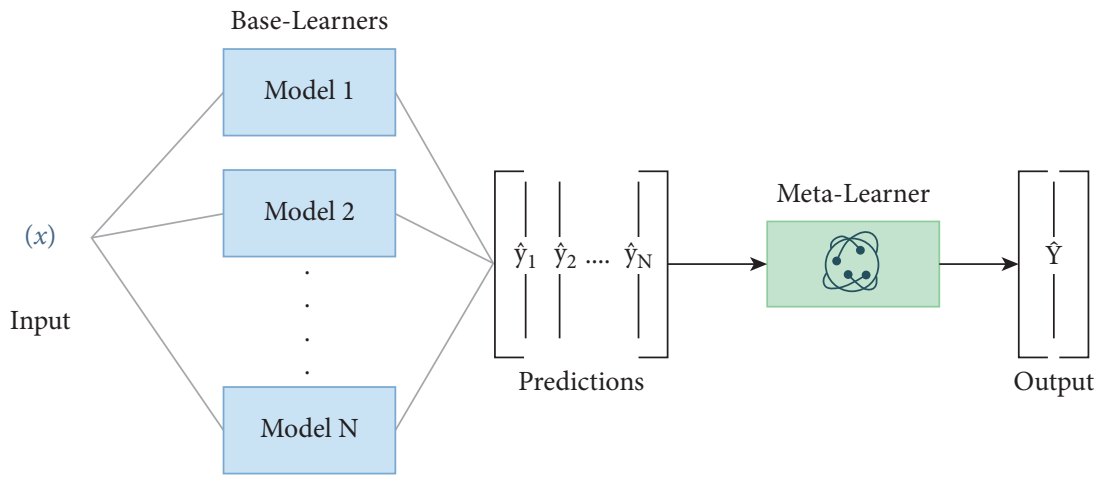

FIGURE 2: Main steps of the stacking technique. 


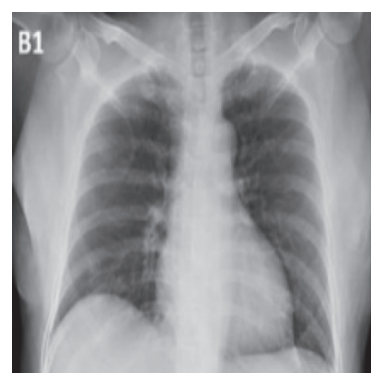

(a)

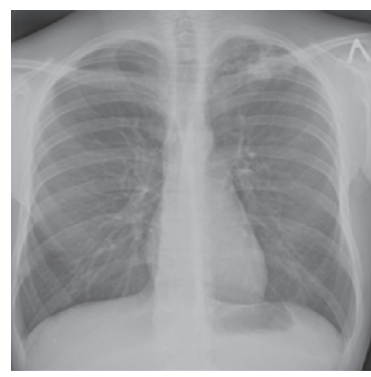

(b)

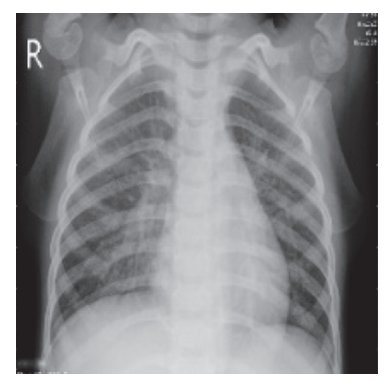

(c)

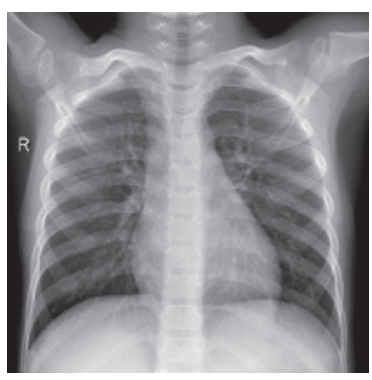

(d)

Figure 3: X-ray image samples of the 4 classes: (a) COVID-19 sample; (b) tuberculosis sample; (c) viral pneumonia sample; (d) normal sample.

TABLE 2: Dataset sources used to generate a combined dataset containing four classes.

\begin{tabular}{|c|c|c|c|c|}
\hline Dataset source & $\begin{array}{l}\text { COVID- } \\
19\end{array}$ & Normal & $\begin{array}{c}\text { Viral } \\
\text { pneumonia }\end{array}$ & Tuberculosis \\
\hline $\begin{array}{l}\text { COVID-19 Radiography Database (https://www.kaggle.com/tawsifurrahman/ } \\
\text { covid19-radiography-database/data?select=COVID-19+Radiography+Database) }\end{array}$ & 1200 & 1341 & 1345 & - \\
\hline $\begin{array}{l}\text { COVID-19 Detection X-Ray Dataset (https://www.kaggle.com/darshan1504/covid19- } \\
\text { detection-xray-dataset) }\end{array}$ & 60 & 880 & 412 & - \\
\hline $\begin{array}{l}\text { COVID-19 Patients Lungs X-Ray Images (https://www.kaggle.com/nabeelsajid917/ } \\
\text { covid-19-x-ray-10000-images?select=dataset) }\end{array}$ & 70 & 28 & - & - \\
\hline $\begin{array}{l}\text { COVID-19 X-Rays (https://www.kaggle.com/andrewmvd/convid19-x-rays? } \\
\text { select=X+rays) }\end{array}$ & 70 & - & - & - \\
\hline $\begin{array}{l}\text { Pneumonia Virus vs Pneumonia Bacteria (https://www.kaggle.com/ } \\
\text { muhammadmasdar/pneumonia-virus-vs-pneumonia-bacteria) }\end{array}$ & 一 & - & 1493 & - \\
\hline $\begin{array}{l}\text { Tuberculosis (TB) Chest X-Ray Database (https://www.kaggle.com/tawsifurrahman/ } \\
\text { tuberculosis-tb-chest-xray-dataset) }\end{array}$ & - & - & - & 3500 \\
\hline Generated dataset & 1400 & 2249 & 3250 & 3500 \\
\hline
\end{tabular}

3.4.3. Tuned MobileNetV2-Based Model. The MobileNetV2 model is a CNN containing 53 layers with a depth of 88 . Developed in 2018 and trained on a million images from the ImageNet database [52], it reached an accuracy of $90.1 \%$ for this dataset. Table 6 represents the model complexity of our proposed tuned architecture.

The architecture consisted of 13665092 total parameters, with 13930680 trainable parameters and 34112 nontrainable parameters.

3.4.4. Tuned VGG16-Based Model. VGG16 is a CNN model proposed by a team of researchers from the University of Oxford. Trained on ImageNet database, the model achieves $90.1 \%$ accuracy. Table 7 illustrates the complexity details of the proposed tuned model.

The architecture consisted of 17469508 total parameters, with 17460508 trainable parameters and 0 nontrainable parameters.

3.4.5. Tuned VGG19-Based Model. VGG19 is a CNN model that was created in 2015. This model is trained on a million images from the ImageNet database with a depth of 26 . It reached an accuracy of $90 \%$ with this dataset. In Table 8 , we present the proposed tuned architecture based on VGG19 model.
The architecture consisted of 22779204 total parameters, with 22779204 trainable parameters and 0 nontrainable parameters.

\section{Results and Discussion}

Before presenting our results and findings, we first present in the following section some common performance evaluation techniques that are usually used to evaluate ML models at training and testing stages. We start by drawing the confusion matrix and calculating some evaluation metrics. This section presents the metrics and the experimental results obtained by studied models. The confusion matrix allows evaluation of the obtained classification.

4.1. Confusion Matrix and Performance Evaluation Measures. In classification problems, a confusion matrix is a table with two dimensions: reference and predicted. This table is used to classify the prediction obtained by classifiers. Moreover, the confusion matrix has identical sets of classes in each row of its dimension. This can allow verifying the confusion between the different classes by calculating four elements, namely, True Positive (TP), False Positive (FP), True Negative (TN), and False Negative (FN) [53]. The confusion elements for each class Class $X$ are given by the following equations: 
TABLe 3: Description of the dataset partitions used in this study.

\begin{tabular}{lcccc}
\hline Class & Training (80\%) & Validation (10\%) & Testing (10\%) & Total (100\%) \\
\hline COVID-19 & 1120 & 140 & 140 & 1400 \\
Tuberculosis & 2800 & 350 & 350 & 3500 \\
Viral pneumonia & 2600 & 325 & 325 & 3250 \\
Normal & 1799 & 225 & 225 & 2249 \\
Total & 8319 & 1040 & 1040 & 10399 \\
\hline
\end{tabular}

$$
\begin{aligned}
& \mathrm{TP}_{\text {ClassX }}=C_{i, i}, \\
& \mathrm{FN}_{\text {ClassX }}=\sum_{l=1}^{4} C_{i, l}-\mathrm{TP}_{\text {ClassX }}, \\
& \mathrm{FP}_{\text {ClassX }}=\sum_{l=1}^{4} C_{l, i}-\mathrm{TP}_{\text {ClassX }}, \\
& \mathrm{TN}_{\text {ClassX }}=\sum_{l=1}^{4} \sum_{k=1}^{4} C_{l, k}-\left(\mathrm{FP}_{\text {ClassX }}+\mathrm{FN}_{\text {ClassX }}+\mathrm{TP}_{\text {ClassX }}\right),
\end{aligned}
$$

where $C_{\mathrm{i}, \mathrm{i}}$ is the number of samples correctly classified for a given class, $C_{\mathrm{i}, 1}$ is the number of negative samples that are confused with another class, $C_{\mathrm{l}, \mathrm{i}}$ is the number of positive samples that are confused with another class, and $C_{\mathrm{l}, \mathrm{k}}$ is the sum of all samples.

We calculated five scoring metrics used in this study: the accuracy, precision, sensitivity, specificity, and negative predictive value (NPV). These metrics are given by the following equations:

$$
\begin{aligned}
\text { accuracy } & =\frac{\mathrm{TP}+\mathrm{TN}}{\mathrm{TP}+\mathrm{TN}+\mathrm{FP}+\mathrm{FN}}, \\
\text { precision } & =\frac{\mathrm{TP}}{\mathrm{TP}+\mathrm{FP}}, \\
\text { sensitivity } & =\frac{\mathrm{TP}}{\mathrm{TP}+\mathrm{FN}}, \\
\text { specificity } & =\frac{\mathrm{TN}}{\mathrm{TN}+\mathrm{FP}}, \\
\mathrm{NPV} & =\frac{\mathrm{TN}}{\mathrm{TN}+\mathrm{FN}} .
\end{aligned}
$$

4.2. Experimental Results. In this section, we present the main experimental results obtained from this study. Firstly, we show the training results by plotting the accuracy and loss curves for all models used. Then, we draw the confusion matrix for each model.

4.2.1. Training of CNN Model Results. In this study, we used the TensorFlow 2.1 library (https://www.tensorflow.org/) to import the original pretrained models and implement the proposed tuned models based on TL technique. We used the library's default Python API. Models were instantiated using the default implementation of Keras (https://github.com/fchollet/keras). Regarding the combination of model performances, we implemented the stacking method in Python language. We used the ScikitLearn Library (https://scikit-learn.org/stable/) for KNN models. All the experiments were executed in Python language, and we used Jupyter library for easy evaluation of the results. Furthermore, we used the online Google Colab platform to train the proposed TL-based models. Note that Google Colab is a cloud service based on Jupyter Notebook for training and researching the algorithms of ML and DL. This platform used Tesla K80 GPU with 12 GB of GDDR5 VRAM, Intel Xeon Processor with 2 cores @ 2.20 GHz and $13 \mathrm{~GB}$ RAM. For all the algorithms used, we performed the training using the Adam optimizer and the cross-entropy loss function. The input image sizes for all arrays are 224-by-224 pixels. Table 9 presents the hyperparameters used to tune the Base-Models used in this study.

We trained all Base-Models, ResNet152V2, ResNet101V2, MobileNetV2, VGG16, and VGG19, across 25 epochs with the same configuration to ensure comparable results. In Figure 4, we present the plots of accuracy and loss function of the five studied classifiers. The plots are drawn for the training and the validation sets of our chest X-ray datasets containing four classes.

Generally, these curves represent epochs on the $x$-axis and improvement on the $y$-axis. The training curve gives an idea about the successful model training. It is computed from the training set. In fact, 25 epochs were sufficient for all the models to converge. The validation curve provides an idea as to whether the model is underfitting, overfitting, or just right for some ranges of hyperparameter values. However, more epochs were needed to reach the convergence stage, especially for VGG19 and VGG16. Moreover, the overfitting degree was weak in all models. Indeed, the convergence of the accuracy on the training set is close to its convergence on the validation set. From these curves, we concluded that all the models reached an accuracy of $98 \%$ during the validation phase. However, the VGG19 model achieves an accuracy value equal to $99.13 \%$. From the loss curves, we noticed that the average loss value for all these models equals $0.1 \%$.

4.2.2. Testing of the Proposed CNN Model Results. The studied models' performances were tested and evaluated using a completely independent data subset already prepared. Before finding the scoring metrics for each performance model, we proceeded with drawing the confusion matrix. Figure 5 represents the confusion matrix of the five trained models. 
TABLE 4: The proposed tuned version based on ResNet152V2 model architecture.

\begin{tabular}{lcc}
\hline Layer type & Output shape & Parameters \\
\hline resnet152v2 (model) & $7 \times 7 \times 2048$ & 58331648 \\
conv2d_1 (Conv2D) & $7 \times 7 \times 1024$ & 2098176 \\
mp_1 (MaxPooling2D) & $3 \times 3 \times 1024$ & 0 \\
flatten (flatten) & 9216 & 0 \\
dense_1 (dense) & 1024 & 9438208 \\
dense_2 (dense) & 512 & 524800 \\
dense_3 (dense) & 256 & 131328 \\
dense_4 (dense) & 4 & 1028 \\
\hline
\end{tabular}

TAвLE 5: The proposed tuned version based on ResNet101V2 model architecture.

\begin{tabular}{lcc}
\hline Layer type & Output shape & Parameters \\
\hline resnet101v2 (model) & $7 \times 7 \times 2048$ & 42626560 \\
conv2d_1 (Conv2D) & $7 \times 7 \times 1024$ & 2098176 \\
mp_1(MaxPooling2D) & $3 \times 3 \times 1024$ & 0 \\
flatten (flatten) & 9216 & 0 \\
dense_1 (dense) & 1024 & 9438208 \\
dense_2 (dense) & 512 & 524800 \\
dense_3 (dense) & 256 & 131328 \\
dense_4 (dense) & 4 & 1028 \\
\hline
\end{tabular}

TABle 6: The proposed tuned MobileNetV2-based model architecture.

\begin{tabular}{lcc}
\hline Layer type & Output shape & Parameters \\
\hline mobilenetv2 (model) & $7 \times 7 \times 1280$ & 2257984 \\
conv2d_1 (Conv2D) & $7 \times 7 \times 1024$ & 1311744 \\
mp_1(MaxPooling2D) & $3 \times 3 \times 1024$ & 0 \\
flatten (flatten) & 9216 & 0 \\
dense_1 (dense) & 1024 & 9438208 \\
dense_2 (dense) & 512 & 524800 \\
dense_3 (dense) & 256 & 131328 \\
dense_4 (dense) & 4 & 1028 \\
\hline
\end{tabular}

TABLE 7: The proposed tuned VGG16-based model architecture.

\begin{tabular}{lcc}
\hline Layer type & Output shape & Parameters \\
\hline vgg16 (model) & $7 \times 7 \times 512$ & 14714688 \\
conv2d_1 (Conv2D) & $7 \times 7 \times 512$ & 262656 \\
mp_1 (MaxPooling2D) & $3 \times 3 \times 512$ & 0 \\
flatten (flatten) & 4608 & 0 \\
dense_1 (dense) & 512 & 2359808 \\
dense_2 (dense) & 256 & 131328 \\
dense_3 (dense) & 4 & 1028 \\
\hline
\end{tabular}

TABLE 8: Complexity of the proposed tuned VGG19-based model architecture.

\begin{tabular}{lcc}
\hline Layer type & Output shape & Parameters \\
\hline vgg19 (model) & $7 \times 7 \times 512$ & 20024384 \\
conv2d_1 (Conv2D) & $7 \times 7 \times 512$ & 262656 \\
mp_1 (MaxPooling2D) & $3 \times 3 \times 512$ & 0 \\
flatten (flatten) & 4608 & 0 \\
dense_1 (dense) & 512 & 2359808 \\
dense_2 (dense) & 256 & 131328 \\
dense_3 (dense) & 4 & 1028 \\
\hline
\end{tabular}


TABLE 9: The best hyperparameters used for the TL models in the training phase.

\begin{tabular}{lccccc}
\hline Network & Learning rate & Batch size & Optimizer & Loss function & Epochs \\
\hline All Base-Models used in this study & $1.000000 \mathrm{e}-04$ & 16 & Adam & Categorical cross entropy & 25
\end{tabular}
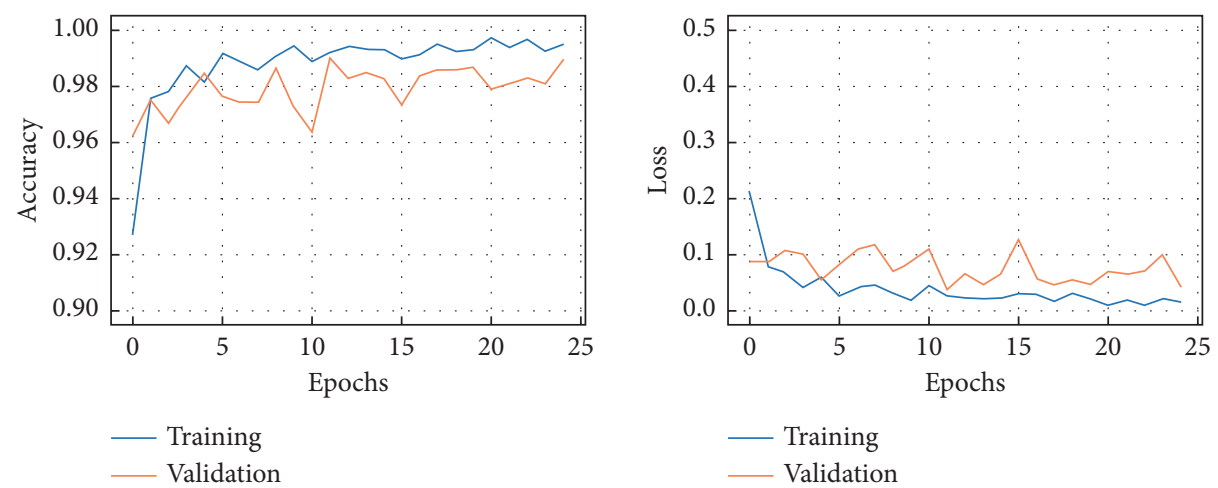

(a)
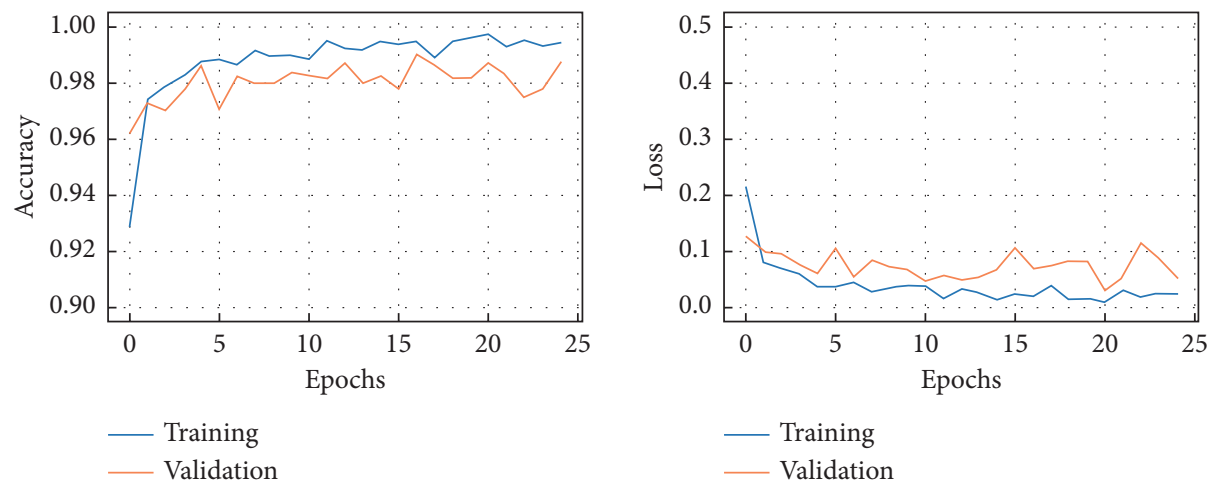

(b)
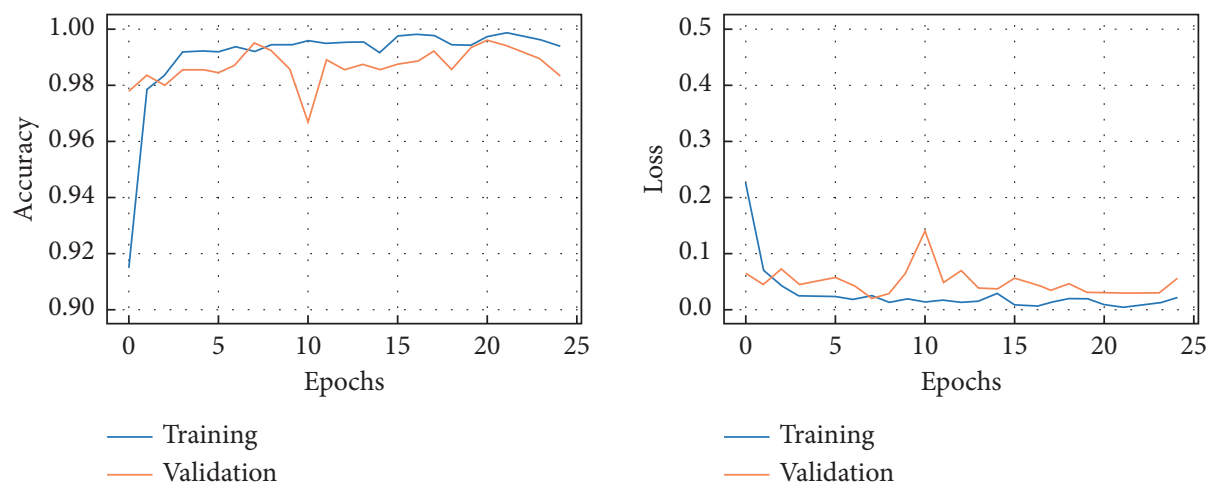

(c)

FIgURe 4: Continued. 

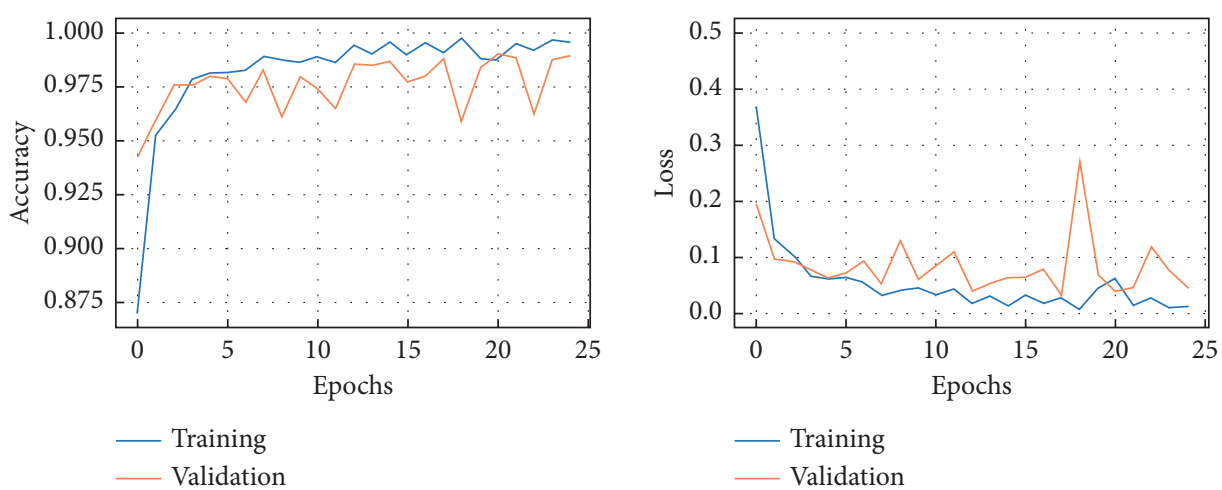

_ Training

Validation

(d)
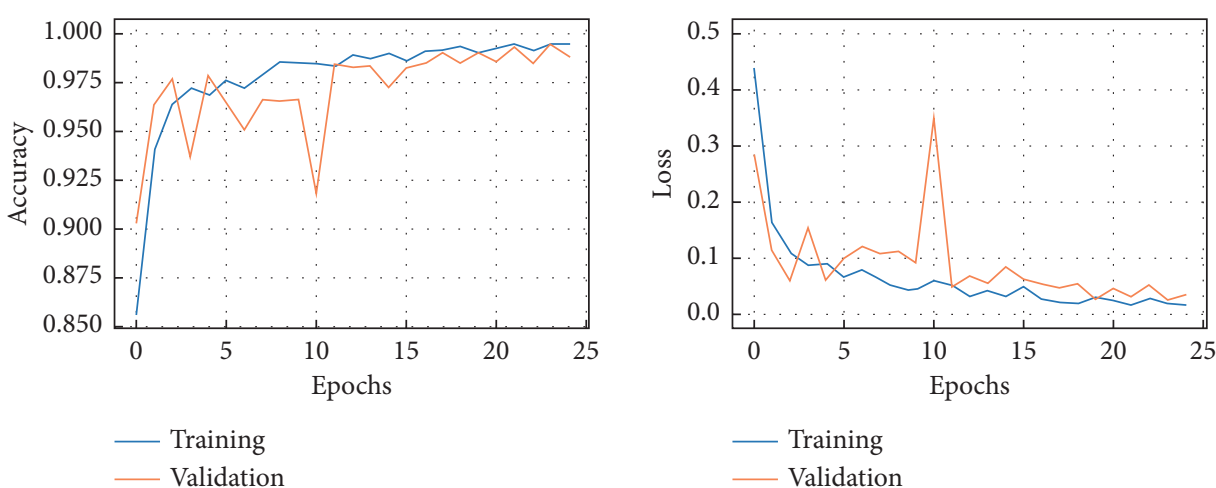

(e)

FiguRE 4: Accuracy and loss curves obtained by training and validation Base-Models: (a) ResNet152V2-based model; (b) ResNet101V2based model; (c) MobileNetV2-based model; (d) VGG16-based model; (e) VGG19-based model.

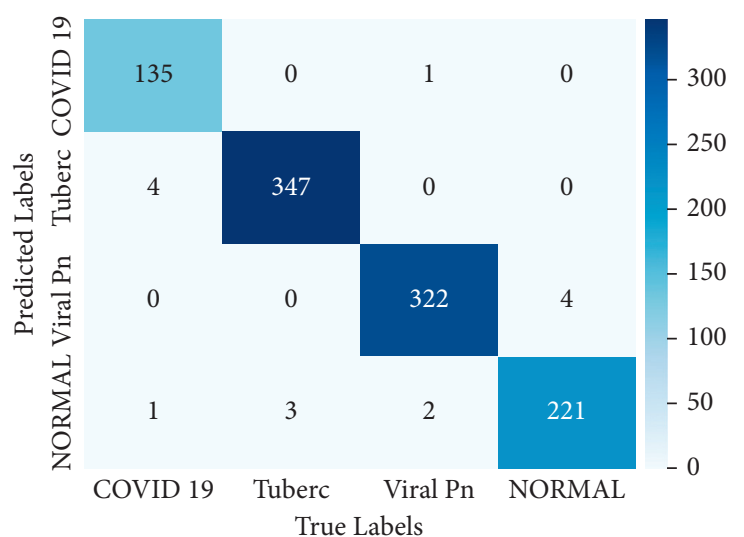

(a)

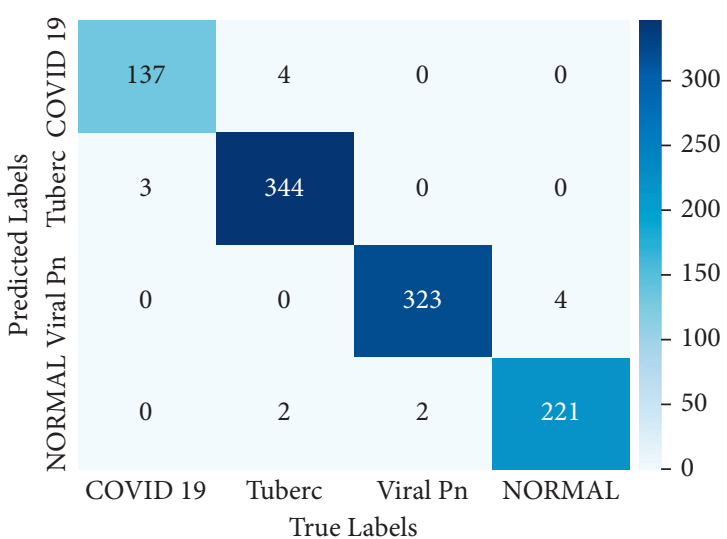

(b)

Figure 5: Continued. 


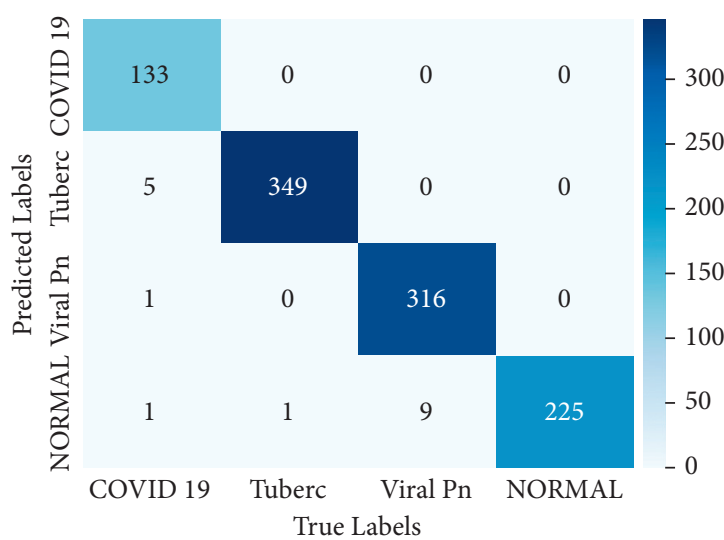

(c)

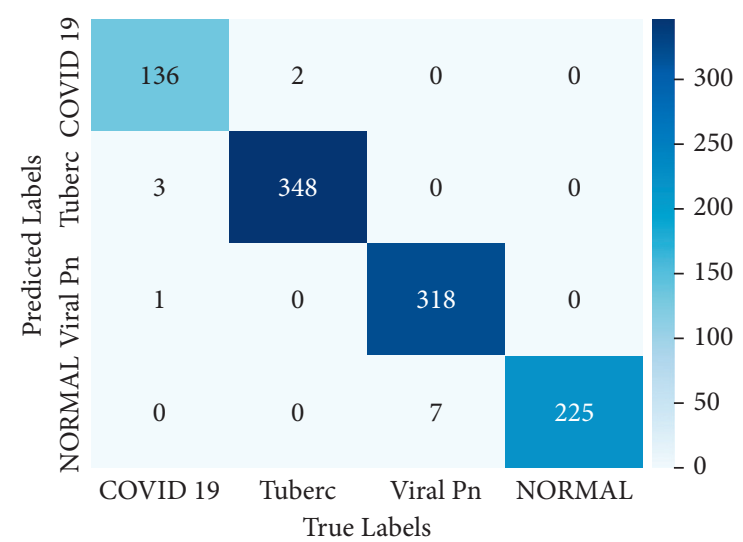

(d)

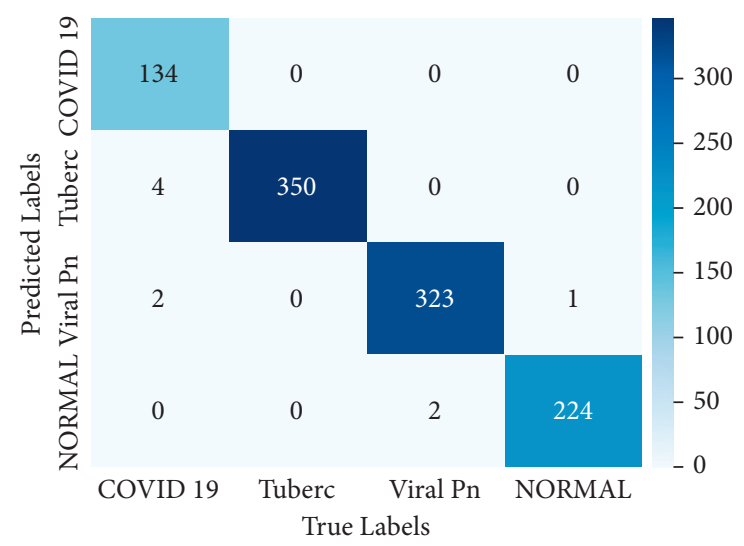

(e)

Figure 5: The confusion matrix representing the Base-Models' classification: (a) ResNet152V2-based model; (b) ResNet101V2-based model; (c) MobileNetV2-based model; (d) VGG16-based model; (e) VGG19-based model.

From these tables, we noticed that the majority of models give a good classification. However, there is some confusion by some models about the classes of COVID-19 and tuberculosis. However, we can see that the majority of models achieve perfect performance for the four classes. Based on these performances, we moved to the second training level by combining the models' classification. In Figure 6, we represent the confusion matrix of the generated model classification using stacking technique. This matrix gives a performance visualization of the generated model. From this classification, we noticed that the TP is higher compared to the FP and FN for all classes. Moreover, we can observe that the FP and FN of three classes, tuberculosis, viral pneumonia, and normal, are larger compared to the COVID-19 class. This model classified correctly 140 cases as COVID-19, and just 4 cases were classified as COVID-19 although they belong to the tuberculosis class. To understand these experimental results, we employed the confusion matrix results to calculate the evaluation metrics for each model.

To explore these results, we can use a ROC curve to plot the sensitivity versus specificity (or False Positive Rate vs True Positive Rate) of a diagnostic test. Generally, this type of curve helps us to compare several models, according to the value of the AUC variable. This value measures the entire area between two dimensions located under the ROC curve. In this paper, we draw the ROC curve for each model used in this study. Figure 7 illustrates a plot of the False Positive Rate (FPR) versus True Positive Rate (TPR) for the different classes for the experimented model and proposed model.

From these ROC curves, we can see that all the studied models reached an AUC value of 0.98 . It becomes clear that the model based on stacking technique is the best model to classify the X-ray images used in this study. Moreover, we noticed that all the classes achieve a rate of area in the range of $0.99-1$. The generated model produced a very high performance compared to the other models. In fact, the proposed model reached an AUC value of 1.00 for COVID-19 class, which has an important clinical advantage.

This means the labeling of COVID-19 cases with other classes is almost zero, which reduces the risk of not detecting COVID-19 cases from their chest X-rays. To clarify these results, Table 10 shows the performance evaluation metrics of the experimented models; the best results are in bold.

From Table 10, we report that most models have a loss value equal to $5.06 \%$ except two models: the VGG16 and generated model achieved a loss value equal to $3.69 \%$ and $3.09 \%$, respectively. Moreover, the generated model obtained the largest values for all performance metrics 


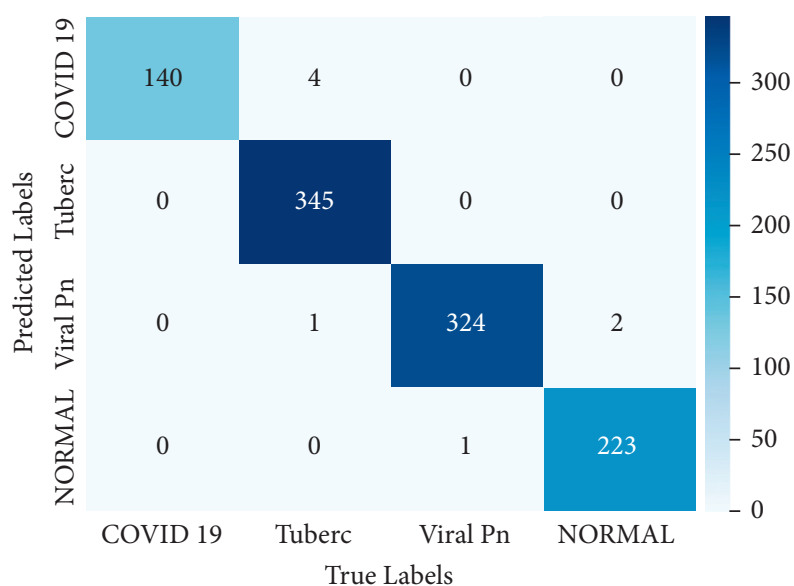

FIGURE 6: The confusion matrix representing the generated model by the stacking technique.
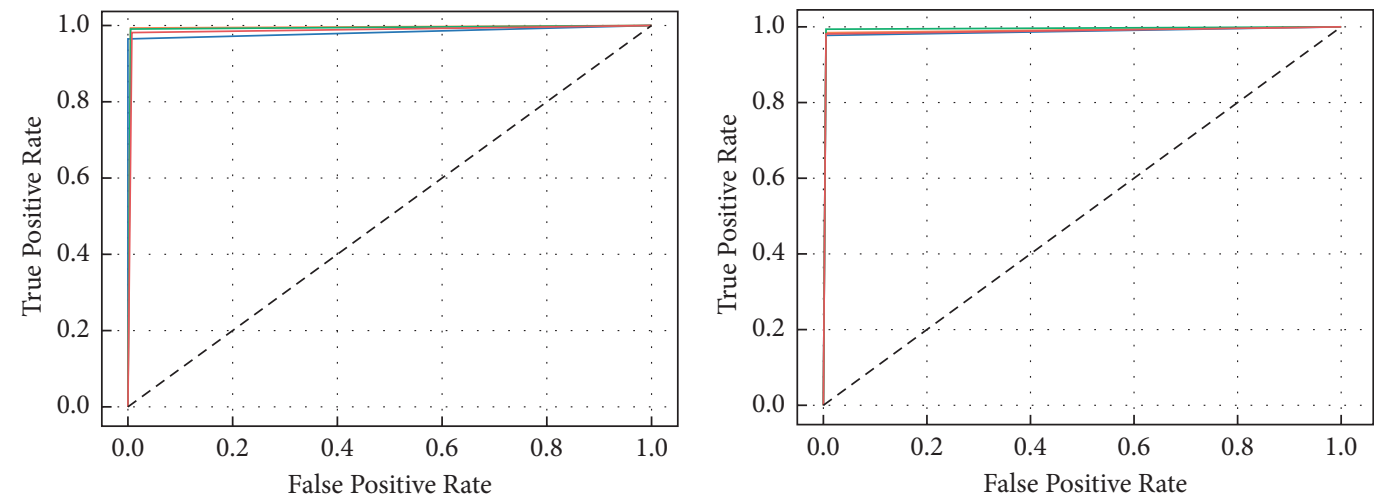

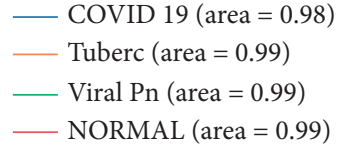

(a)

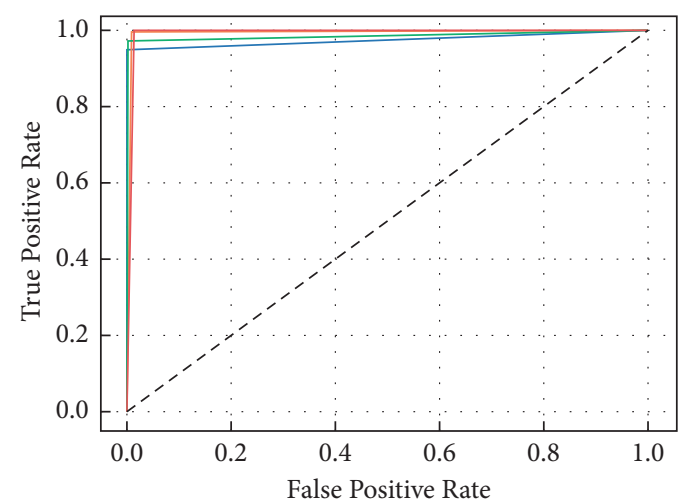

COVID $19($ area $=0.97)$

Tuberc $($ area $=0.99)$

Viral Pn $($ area $=0.99)$

NORMAL $($ area $=0.99)$

(c)
COVID $19($ area $=0.99)$

Tuberc $($ area $=0.99)$

— Viral Pn $($ area $=0.99)$

NORMAL $($ area $=0.99)$

(b)

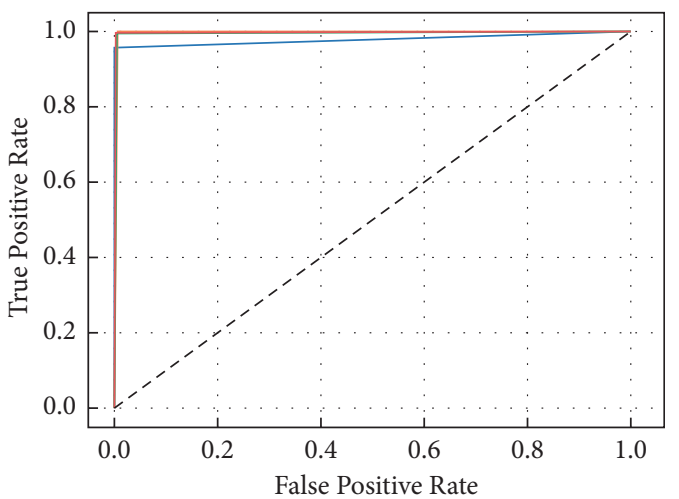

- COVID $19($ area $=0.98)$

Tuberc $($ area $=1.00)$

Viral Pn $($ area $=0.99)$

NORMAL $($ area $=1.00)$

(d)

Figure 7: Continued. 


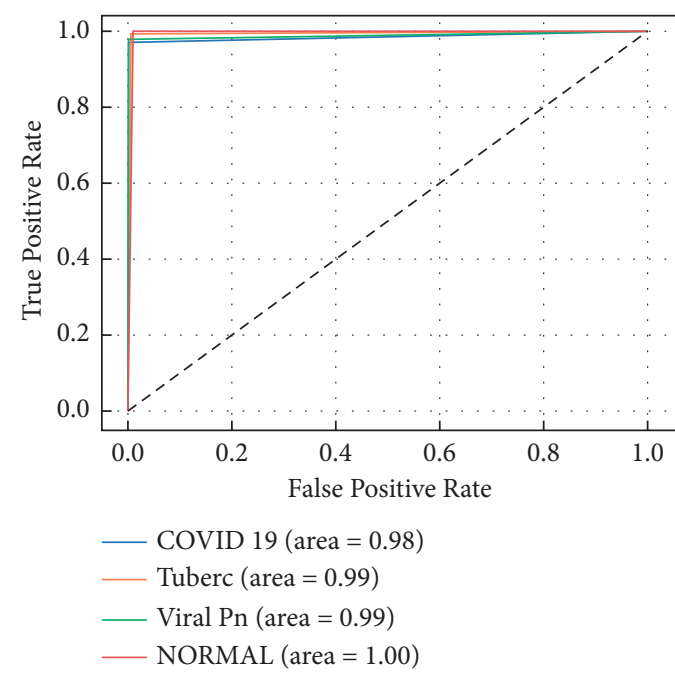

(e)

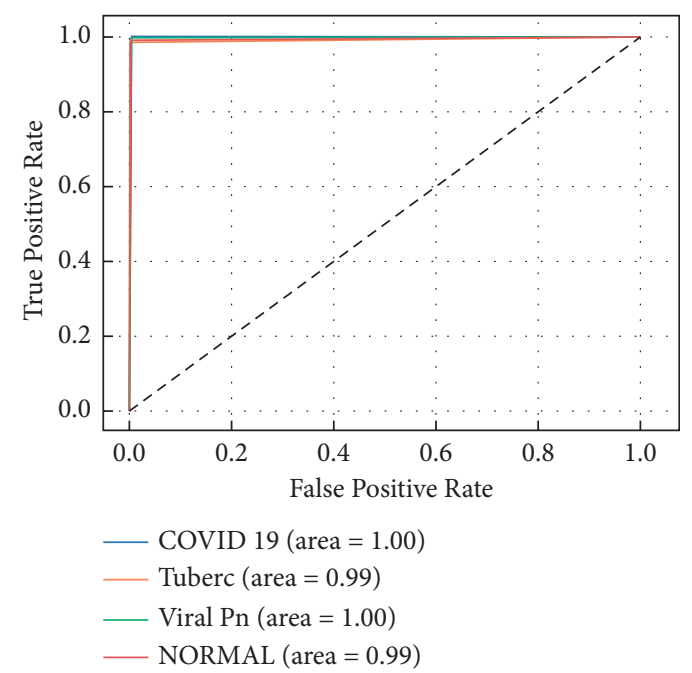

(f)

FIGURE 7: ROC curves results for 5 tuned CNN models: (a) ResNet152V2-based model; (b) ResNet101V2-based model; (c) MobileNetV2based model; (d) VGG19-based model; (e) VGG16-based model. (f) The resulting proposed CNN model obtained by the stacking technique.

TABLE 10: Models' performance evaluation based on scoring metrics: loss, accuracy, precision, sensitivity, specificity, and NPV.

\begin{tabular}{|c|c|c|c|c|c|c|}
\hline Model & Accuracy (\%) & Loss (\%) & Precision (\%) & Sensitivity (\%) & Specificity (\%) & NPV (\%) \\
\hline ResNet152V2 & 98.55 & 5.06 & 98.56 & 98.22 & 99.5 & 99.52 \\
\hline ResNet101V2 & 98.55 & 5.99 & 98.32 & 98.44 & 99.52 & 99.51 \\
\hline MobileNetV2 & 98.36 & 5.18 & 98.4 & 97.99 & 99.45 & 99.46 \\
\hline VGG16 & 98.75 & 3.69 & 98.59 & 98.6 & 99.59 & 99.57 \\
\hline VGG19 & 99.13 & 5.51 & 99.27 & 98.66 & 99.69 & 99.73 \\
\hline Generated model by stacking technique & 99.23 & 3.09 & 98.96 & 99.34 & 99.75 & 99.72 \\
\hline
\end{tabular}

Table 11: Description of Base-Models' runtime and time by epoch.

\begin{tabular}{lcccc}
\hline Models & Accuracy $(\%)$ & Runtime & Time/epoch (s) & Total parameters (millions) \\
\hline ResNet152V2 & 98.55 & $50 \min 33 \mathrm{~s}$ & 120 & 70 \\
ResNet101V2 & 98.55 & $35 \min 21 \mathrm{~s}$ & 85 & 54 \\
MobileNetV2 & 98.36 & $19 \min 50 \mathrm{~s}$ & 47 & 13 \\
VGG16 & 98.75 & $24 \min 27 \mathrm{~s}$ & 59 & 17 \\
VGG19 & 99.13 & $28 \min 55 \mathrm{~s}$ & 69 & 22 \\
\hline
\end{tabular}

computed. This model records the lowest loss value equal to $3.09 \%$. On the other hand, we noticed that the four classifiers ResNet152V2, ResNet101V2, MobileNetV2, and VGG16 reached an accuracy of $98 \%$. However, the VGG19 and the generated model achieved an accuracy of $99.13 \%$ and 99.23\%, respectively. For the NPV metric, the Base-Models and the proposed model improve a high value of $99.5 \%$.

4.2.3. Runtime Results. The runtime is an important parameter improving the efficiency and the reliability of the system. We compared the required time during the training process of the experimented models. From Table 11, we observe the approximate change in time at the training phase from one model to another. This is mainly due to the total number of parameters for each model. Moreover, when the number of model parameters is high, the time required for the training phase became longer, whether the runtime or the time necessary for each epoch.

4.3. Discussion. In this paper, we proposed a novel diagnosis system of COVID-19 based on the stacking technique and TL algorithms. This system aims to find the best diagnostic algorithm for patients infected with COVID-19. The generated model was based on the five TL networks: ResNet152V2, ResNet101V2, MobileNetV2, VGG16, and VGG19. These algorithms were trained and validated on the generated X-ray image dataset from a six-source database. This dataset includes four classes: COVID-19, tuberculosis, viral pneumonia, and normal. At the metamodel level, we used a KNN algorithm to generate a final predictive model. 


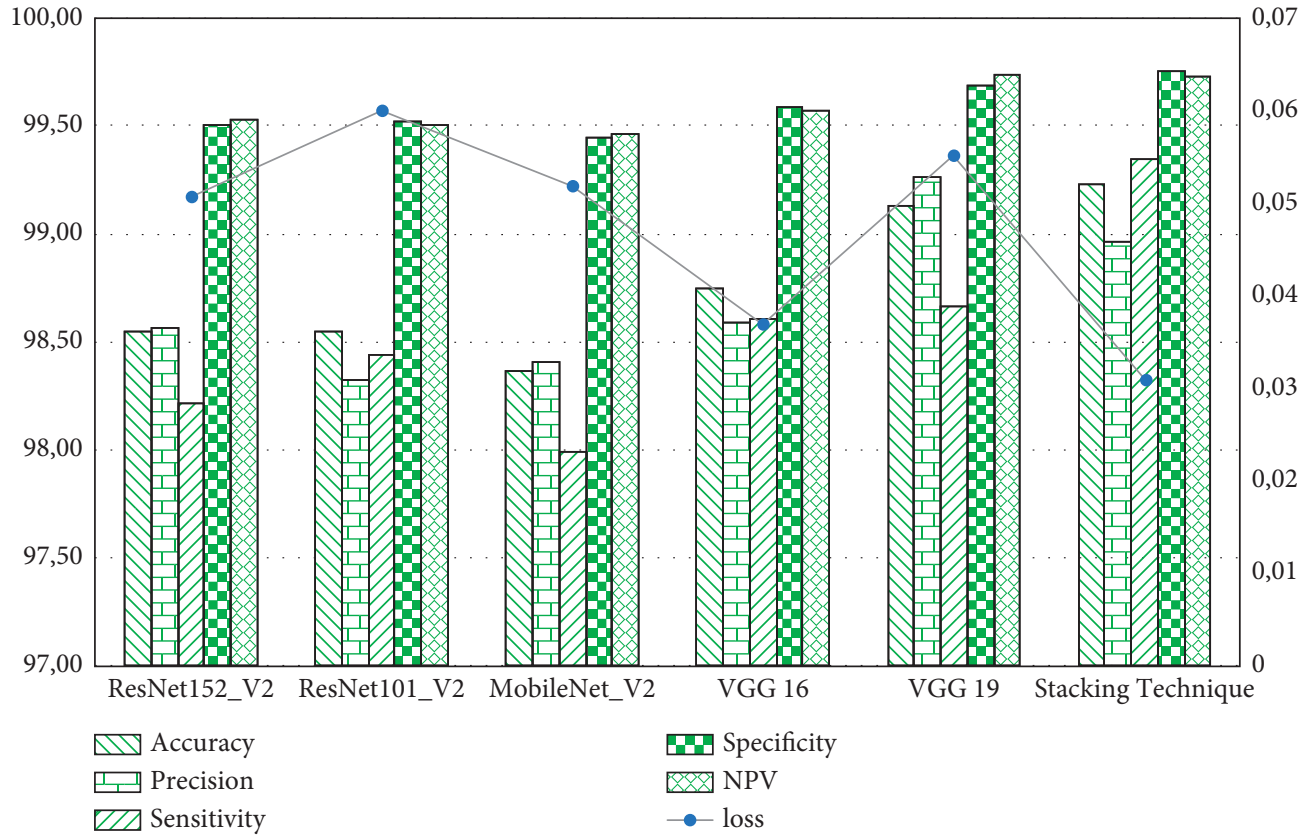

FIGURE 8: The performance evaluation metrics for all studied models.

TABLE 12: Results for the staking technique-based model compared with some previous works.

\begin{tabular}{|c|c|c|c|c|c|}
\hline Works & Methods used & Accuracy & Precision & Sensitivity & Specificity \\
\hline [35] & ResNet50 & $93.01 \%$ & $95.18 \%$ & $91.45 \%$ & $94.77 \%$ \\
\hline \multirow{2}{*}{ [36] } & \multirow{2}{*}{ CovXNet } & $89.6 \%$ & $88.5 \%$ & $90.3 \%$ & $87.6 \%$ \\
\hline & & $90.2 \%$ & $90.8 \%$ & $89.9 \%$ & $89.1 \%$ \\
\hline \multirow{2}{*}{ [37] } & \multirow{2}{*}{ CoroNet } & $95 \%$ & $95 \%$ & $96.9 \%$ & $97.5 \%$ \\
\hline & & $89.6 \%$ & $90 \%$ & $89.92 \%$ & $96.4 \%$ \\
\hline \multirow{10}{*}{ [38] } & AlexNet & $78.92 \%$ & $\mathrm{~N} / \mathrm{A}$ & $89.21 \%$ & $68.63 \%$ \\
\hline & VGG16 & $83.33 \%$ & $\mathrm{~N} / \mathrm{A}$ & $80.39 \%$ & $86.27 \%$ \\
\hline & VGG19 & $85.29 \%$ & $\mathrm{~N} / \mathrm{A}$ & $92.16 \%$ & $78.43 \%$ \\
\hline & SqueezeNet & $82.84 \%$ & $\mathrm{~N} / \mathrm{A}$ & $78.43 \%$ & $87.52 \%$ \\
\hline & GoogLeNet & $85.29 \%$ & $\mathrm{~N} / \mathrm{A}$ & $81.37 \%$ & $90.20 \%$ \\
\hline & MobileNetV2 & $92.16 \%$ & N/A & $97.06 \%$ & $87.25 \%$ \\
\hline & ResNet18 & $91.61 \%$ & $\mathrm{~N} / \mathrm{A}$ & $95.10 \%$ & $88.23 \%$ \\
\hline & ResNet50 & $94.12 \%$ & $\mathrm{~N} / \mathrm{A}$ & $90.20 \%$ & $100 \%$ \\
\hline & ResNet101 & $99.51 \%$ & N/A & $100 \%$ & $99.02 \%$ \\
\hline & Xception & $99.02 \%$ & $\mathrm{~N} / \mathrm{A}$ & $98.04 \%$ & $100 \%$ \\
\hline \multirow{5}{*}{ [39] } & VGG16 & $79.01 \%$ & $\mathrm{~N} / \mathrm{A}$ & N/A & $\mathrm{N} / \mathrm{A}$ \\
\hline & DenseNet 121 & $89.96 \%$ & $\mathrm{~N} / \mathrm{A}$ & $\mathrm{N} / \mathrm{A}$ & $\mathrm{N} / \mathrm{A}$ \\
\hline & Xception & $88.03 \%$ & $\mathrm{~N} / \mathrm{A}$ & $\mathrm{N} / \mathrm{A}$ & $\mathrm{N} / \mathrm{A}$ \\
\hline & NASNet & $85.03 \%$ & $\mathrm{~N} / \mathrm{A}$ & $\mathrm{N} / \mathrm{A}$ & $\mathrm{N} / \mathrm{A}$ \\
\hline & EfficientNet & $93.48 \%$ & $\mathrm{~N} / \mathrm{A}$ & N/A & N/A \\
\hline$[40]$ & AlexNet & $99.13 \%$ & $\mathrm{~N} / \mathrm{A}$ & $99.47 \%$ & $99.15 \%$ \\
\hline$[41]$ & $\begin{array}{l}\text { Majority voting } \\
\text { method }\end{array}$ & $99.31 \%$ & $100 \%$ & $100 \%$ & N/A \\
\hline$[42]$ & DenseNet & $97.99 \%$ & $98.38 \%$ & $98.15 \%$ & $\mathrm{~N} / \mathrm{A}$ \\
\hline \multirow[t]{2}{*}[43]{} & $\begin{array}{l}\text { Majority voting } \\
\text { method }\end{array}$ & $99.26 \%$ & $97.87 \%$ & $100 \%$ & $98.89 \%$ \\
\hline & ResNet50V2 & $95.49 \%$ & $96.85 \%$ & $99.19 \%$ & $98.27 \%$ \\
\hline \multirow[t]{2}{*}{ [44] } & VGG16 & $92.70 \%$ & $97.50 \%$ & $94.35 \%$ & $98.69 \%$ \\
\hline & Inception V3 & $92.97 \%$ & $97.60 \%$ & $98.39 \%$ & $98.67 \%$ \\
\hline \multirow[t]{2}{*}{ [45] } & VGG16 & $91.69 \%$ & $92.33 \%$ & $95.92 \%$ & $100 \%$ \\
\hline & VGG16 & $87.84 \%$ & $82.00 \%$ & $82.33 \%$ & $91.20 \%$ \\
\hline \multirow[t]{2}{*}{ [46] } & Inception V3 & $91.32 \%$ & $87.54 \%$ & $89.00 \%$ & $94.00 \%$ \\
\hline & EfficientNetB0 & $92.93 \%$ & $88.30 \%$ & $90.00 \%$ & $95.00 \%$ \\
\hline $\begin{array}{l}\text { Proposed } \\
\text { model }\end{array}$ & Stacking technique & $\begin{array}{c}99.23 \%[95 \% \mathrm{CI}: \\
98.3-100]\end{array}$ & $\begin{array}{c}98.96 \%[95 \% \text { CI: } \\
98-100]\end{array}$ & $\begin{array}{c}99.34 \%[95 \% \mathrm{CI}: \\
98.4-100]\end{array}$ & $\begin{array}{c}99.75 \%[95 \% \text { CI: } \\
98.5-100]\end{array}$ \\
\hline
\end{tabular}

The bold values mean the performance evaluation metrics obtained with our proposed model based on stacking technique. The values between [ ] lead to the confidence interval (CI) that is the standard used to quantify the uncertainty of estimating the obtained performance evaluation metrics. 
In fact, the KNN algorithm learns how to combine the basic models' predictions and provide the final prediction of patients infected with COVID-19. From the experimental results, we noticed that all the studied models achieve a high accuracy between $98 \%$ and $99 \%$. Furthermore, the loss value for most models does not exceed 5\%. The graphic presented in Figure 8 summarizes all experimental results presented in this paper.

This graph shows the variation between loss and three scoring metrics: accuracy, precision, and sensitivity. Indeed, when the loss value increases, the values of the three metrics decrease. All models used in this work including the generated model achieved a high value in specificity and NPV metrics. Generally, the proposed diagnostic system showed a high performance compared to other previous works. Table 12 illustrates a comparison between our proposed system and the other works presented in this paper.

The PCR test is considered by many to be the gold standard for diagnosing COVID-19. Calculating the concordance rate between our method and the PCR test allows us to better judge the potential of our system for prevalent and widespread adoption in the real state of the COVID-19 pandemic. In particular, our system is able to distinguish between four classes: COVID-19, tuberculosis, viral pneumonia, and normal.

\section{Conclusion and Perspectives}

The main contribution of this paper is to propose an efficient pandemic disease diagnostic system. We targeted the COVID-19 diagnostic task from chest X-ray images. The proposed system is based on five basic transfer learning models. Furthermore, the goal was to improve the detection precision of COVID-19 by proposing a new diagnostic tool that combines the performance of TL algorithms to extract the images' features. This allows more stable predictions to be made and improves the learning model. We started by preparing the dataset to be used. We selected the best tested deep learning models from the current state-of-the-art image classification algorithms. We developed their architecture to add our designed head model. We trained all selected classifiers on the processed dataset. We found very encouraging results when testing the test set. All classifiers have an accuracy of about $99 \%$.

To go beyond improving accuracy, we selected the best performing classifiers on the test set. To reinforce our results, we performed the experiments on two different sets (the test set and the validation set). We have found that the best approach to take for COVID-19 diagnosis is the stacking method based on the results given by the studied classifiers: ResNet152V2, ResNet101V2, MobileNetV2, VGG16, and VGG19. The stacking method gave us an average accuracy of 0.9923 with $100 \%$ accuracy regarding the COVID-19 class when testing on the test and validation set.

This study places more emphasis on the usefulness of the stacking method in dealing with sensitive and important tasks, such as diagnosing COVID-19.

In future work, we need to invest more in voting approaches by studying their performance on larger datasets.
Moreover, we will implement the three ensemble ML methods, using bagging, boosting, and stacking technique. We need to dig deeper into the use of a multilevel stacking technique, to make our system more robust and accurate for diagnosing pandemic or cancerous diseases.

\section{Data Availability}

The data used to support the results are available from the corresponding author upon reasonable request.

\section{Conflicts of Interest}

The authors declare that they have no conflicts of interest regarding the publication of this work.

\section{Authors' Contributions}

S.H., O.E.G, B.C, and H.M conceptualized the study, prepared methodology, arranged software, done formal analysis, investigated the project, and wrote and prepared the original draft; B.C., A.R., and H.O. supervised and reviewed and edited the manuscript. All the authors have read and agreed to the published version of the manuscript.

\section{Acknowledgments}

The authors are thankful to the Centre National pour la Recherche Scientifique et Technique (CNRST) and the Hassan II University of Casablanca, Morocco, for their support to this work as a part of a project entitled "Scientific and Technological Research Support Program in Link with COVID-19" launched in April 2020 (reference: Letter to the Director of "Ecole Normale Supérieure de l'Enseignement Technique de Mohammedia” dated June 10, 2020).

\section{References}

[1] V. Izda, M. A. Jeffries, and A. H. Sawalha, "COVID-19: a review of therapeutic strategies and vaccine candidates," Clinical Immunology, vol. 222, Article ID 108634, 2021.

[2] R. Chakraborty and S. Parvez, "COVID-19: an overview of the current pharmacological interventions, vaccines, and clinical trials," Biochemical Pharmacology, vol. 180, Article ID 114184, 2020.

[3] M. A. Alzubaidi, M. Otoom, N. Otoum, Y. Etoom, and R. Banihani, "A novel computational method for assigning weights of importance to symptoms of COVID-19 patients," Artificial Intelligence in Medicine, vol. 112, Article ID 102018, 2021.

[4] M. Tandan, Y. Acharya, S. Pokharel, and M. Timilsina, "Discovering symptom patterns of COVID-19 patients using association rule mining," Computers in Biology and Medicine, vol. 131, Article ID 104249, 2021.

[5] S. Hamida, B. Cherradi, O. Terrada, A. Raihani, H. Ouajji, and S. Laghmati, "A novel feature extraction system for cursive word vocabulary recognition using local features descriptors and gabor filter," in Proceedings of the 2020 3rd International Conference on Advanced Communication Technologies and Networking (CommNet), pp. 1-7, Marrakech, Morocco, September 2020. 
[6] A. Alsaeedi and M. Al-Sarem, "Detecting rumors on social media based on a CNN deep learning technique," Arabian Journal for Science and Engineering, vol. 45, no. 12, pp. 10813-10844, 2020.

[7] A. K. M. N. Islam, S. Laato, S. Talukder, and E. Sutinen, "Misinformation sharing and social media fatigue during COVID-19: an affordance and cognitive load perspective," Technological Forecasting and Social Change, vol. 159, Article ID 120201, 2020.

[8] O. Terrada, S. Hamida, B. Cherradi, A. Raihani, and O. Bouattane, "Supervised machine learning based medical diagnosis support system for prediction of patients with heart disease," Advances in Science, Technology and Engineering Systems Journal, vol. 5, no. 5, pp. 269-277, 2020.

[9] T. Li, W. Wei, L. Cheng et al., "Computer-aided diagnosis of COVID-19 CT scans based on spatiotemporal information fusion," Journal of Healthcare Engineering, vol. 2021, Article ID 6649591, 11 pages, 2021.

[10] B. Cherradi, O. Terrada, A. Ouhmida, S. Hamida, A. Raihani, and O. Bouattane, "Computer-aided diagnosis system for early prediction of atherosclerosis using machine learning and K-fold cross-validation," in Proceedings of the 2021 International Congress of Advanced Technology and Engineering (ICOTEN), pp. 1-9, Taiz, Yemen, July 2021.

[11] O. Terrada, B. Cherradi, S. Hamida, A. Raihani, H. Moujahid, and O. Bouattane, "Prediction of patients with heart disease using artificial neural network and adaptive boosting techniques," in Proceedings of the 2020 3rd International Conference on Advanced Communication Technologies and Networking (CommNet), pp. 1-6, Marrakech, Morocco, September 2020.

[12] O. Terrada, B. Cherradi, A. Raihani, and O. Bouattane, "A fuzzy medical diagnostic support system for cardiovascular diseases diagnosis using risk factors," in Proceedings of the 2018 International Conference on Electronics, Control, Optimization and Computer Science (ICECOCS), pp. 1-6, Kenitra, December 2018.

[13] O. Terrada, B. Cherradi, A. Raihani, and O. Bouattane, "Classification and prediction of atherosclerosis diseases using machine learning algorithms," in Proceedings of the 2019 5th International Conference on Optimization and Applications (ICOA), pp. 1-5, Kenitra, Morocco, April 2019.

[14] O. Terrada, B. Cherradi, A. Raihani, and O. Bouattane, "A novel medical diagnosis support system for predicting patients with atherosclerosis diseases," Informatics in Medicine Unlocked, vol. 21, Article ID 100483, 2020.

[15] N. A. Ali, B. Cherradi, A. El Abbassi, O. Bouattane, and M. Youssfi, "New parallel hybrid implementation of bias correction fuzzy C-means algorithm," in Proceedings of the 2017 International Conference on Advanced Technologies for Signal and Image Processing (ATSIP), pp. 1-6, Fez, May 2017.

[16] N. Ait Ali, B. Cherradi, A. El Abbassi, O. Bouattane, and M. Youssfi, "GPU fuzzy c-means algorithm implementations: performance analysis on medical image segmentation," Multimedia Tools and Applications, vol. 77, no. 16, pp. 21221-21243, 2018.

[17] O. Bouattane, B. Cherradi, M. Youssfi, and M. O. Bensalah, "Parallel c-means algorithm for image segmentation on a reconfigurable mesh computer," Parallel Computing, vol. 37, no. 4-5, pp. 230-243, 2011.

[18] S. Sara, B. Samir, H. Ahmed, and C. Bouchaib, "A robust comparative study of five brain extraction algorithms (BET; BSE; McStrip; SPM2; TMBE)," in Proceedings of the 2014
Second World Conference on Complex Systems (WCCS), pp. 632-636, Agadir, Morocco, November 2014.

[19] S. T. Yassine, S. Sara, C. Bouchaib, and R. Abdelilah, "A new fast brain tumor extraction method based on Nl-means and expectation maximization," in Proceedings of the 2018 4th International Conference on Optimization and Applications (ICOA), pp. 1-5, Mohammedia, April 2018.

[20] N. Aitali, B. Cherradi, O. Bouattane, M. Youssfi, and A. Raihani, "New fine-grained clustering algorithm on GPU architecture for bias field correction and MRI image segmentation," in Proceedings of the 2015 27th International Conference on Microelectronics (ICM), pp. 118-121, Casablanca, Morocco, December 2015.

[21] S. Sandabad, A. Hammouch, Y. Sayed Tahri, A. Benba, and B. Cherradi, "New brain extraction method using expectation maximization and mathematical morphology," Journal of Theoretical and Applied Information Technology, vol. 73, no. 3, pp. 368-376, 2015.

[22] S. Laghmati, A. Tmiri, and B. Cherradi, "Machine learning based system for prediction of breast cancer severity," in Proceedings of the 2019 International Conference on Wireless Networks and Mobile Communications (WINCOM), pp. 1-5, Fez, Morocco, October 2019.

[23] O. El Gannour, S. Hamida, B. Cherradi, A. Raihani, and H. Moujahid, "Performance evaluation of transfer learning technique for automatic detection of patients with COVID-19 on X-Ray images," in Proceedings of the 2020 IEEE 2nd International Conference on Electronics, Control, Optimization and Computer Science (ICECOCS), pp. 1-6, Kenitra, Morocco, December 2020.

[24] H. C. Yalcin and A. Kaushik, "Support of intelligent emergent materials to combat COVID-19 pandemic," Emergent Materials, vol. 4 , no. 1, pp. 1-2, 2021.

[25] B. K. Umri, M. Wafa Akhyari, and K. Kusrini, "Detection of Covid-19 in Chest X-ray image using CLAHE and convolutional neural network," in Proceedings of the 2020 2nd International Conference on Cybernetics and Intelligent System (ICORIS), pp. 1-5, Manado, Indonesia, October 2020.

[26] H. Moujahid, B. Cherradi, M. Al-Sarem, and L. Bahatti, "Diagnosis of COVID-19 disease using convolutional neural network models based transfer learning," in Innovative Systems for Intelligent Health Informatics, F. Saeed, F. Mohammed, and A. Al-Nahari, Eds., vol. 72Springer International Publishing, Lecture Notes on Data Engineering and Communications Technologies, , pp. 148-159, 2021.

[27] X. Li, W. Tan, P. Liu, Q. Zhou, and J. Yang, "Classification of COVID-19 chest CT images based on ensemble deep learning," Journal of Healthcare Engineering, vol. 2021, Article ID 5528441, 7 pages, 2021.

[28] M. Toğaçar, B. Ergen, and Z. Cömert, "COVID-19 detection using deep learning models to exploit social mimic optimization and structured chest X-ray images using fuzzy color and stacking approaches," Computers in Biology and Medicine, vol. 121, Article ID 103805, 2020.

[29] S. Tabik, A. Gomez-Rios, J. L. Martin-Rodriguez et al., "COVIDGR dataset and COVID-SDNet methodology for predicting COVID-19 based on chest X-ray images," IEEE Journal of Biomedical and Health Informatics, vol. 24, no. 12, pp. 3595-3605, 2020.

[30] Y. Jiang, H. Chen, M. Loew, and H. Ko, "COVID-19 CT image synthesis with a conditional generative adversarial network," IEEE Journal of Biomedical and Health Informatics, vol. 25, no. 2, pp. 441-452, 2021. 
[31] D. C. d. S. Gomes and G. L. d. O. Serra, "Machine learning model for computational tracking and forecasting the COVID-19 Dynamic Propagation," IEEE Journal of Biomedical and Health Informatics, vol. 25, no. 3, pp. 615-622, 2021.

[32] S. Hamida, O. E. Gannour, B. Cherradi, H. Ouajji, and A. Raihani, "Optimization of machine learning algorithms hyper-parameters for improving the prediction of patients infected with COVID-19," in Proceedings of the 2020 IEEE 2nd International Conference on Electronics, Control, Optimization and Computer Science (ICECOCS), pp. 1-6, Kenitra, Morocco, December 2020.

[33] C. C. John, V. Ponnusamy, S. Krishnan Chandrasekaran, and N. R, "A survey on mathematical, machine learning and deep learning models for COVID-19 transmission and diagnosis," IEEE Reviews in Biomedical Engineering, vol. 2021, Article ID 3069213, 1 page, 2021.

[34] E. F. Ohata, G. M. Bezerra, J. V. S. d. Chagas et al., "Automatic detection of COVID-19 infection using chest X-ray images through transfer learning," IEEE/CAA Journal of Automatica Sinica, vol. 2020, Article ID 1003393, 10 pages, 2020.

[35] Y. Pathak, P. K. Shukla, A. Tiwari, S. Stalin, S. Singh, and P. K. Shukla, "Deep transfer learning based classification model for COVID-19 disease," IRBM, vol. 2020, Article ID S1959031820300993, 2020.

[36] T. Mahmud, M. A. Rahman, and S. A. Fattah, "CovXNet: a multi-dilation convolutional neural network for automatic COVID-19 and other pneumonia detection from chest X-ray images with transferable multi-receptive feature optimization," Computers in Biology and Medicine, vol. 122, Article ID 103869, 2020.

[37] A. I. Khan, J. L. Shah, and M. M. Bhat, "CoroNet: a deep neural network for detection and diagnosis of COVID-19 from chest x-ray images," Computer Methods and Programs in Biomedicine, vol. 196, Article ID 105581, 2020.

[38] A. A. Ardakani, A. R. Kanafi, U. R. Acharya, N. Khadem, and A. Mohammadi, "Application of deep learning technique to manage COVID-19 in routine clinical practice using CT images: results of 10 convolutional neural networks," Computers in Biology and Medicine, vol. 121, Article ID 103795, 2020.

[39] B. Nigam, A. Nigam, R. Jain, S. Dodia, N. Arora, and B. Annappa, "COVID-19: automatic detection from X-ray images by utilizing deep learning methods," Expert Systems with Applications, vol. 176, Article ID 114883, 2021.

[40] M. Kaur, V. Kumar, V. Yadav, D. Singh, N. Kumar, and N. N. Das, "Metaheuristic-based deep COVID-19 screening model from chest X-ray images," Journal of Healthcare Engineering, vol. 2021, Article ID 8829829, 9 pages, 2021.

[41] M. Ben Jabra, A. Koubaa, B. Benjdira, A. Ammar, and H. Hamam, "COVID-19 diagnosis in chest X-rays using deep learning and majority voting," Applied Sciences, vol. 11, no. 6, Article ID 2884, 2021.

[42] F. J. P. Montalbo, "Diagnosing covid-19 chest X-rays with a lightweight truncated densenet with partial layer freezing and feature fusion," Biomedical Signal Processing and Control, vol. 68, Article ID 102583, 2021.

[43] M. Shorfuzzaman and M. Masud, "On the detection of COVID-19 from chest X-ray images using cnn-based transfer learning," Computers, Materials \& Continua, vol. 64, no. 3, pp. 1359-1381, 2020.

[44] M. Shorfuzzaman, M. Masud, H. Alhumyani, D. Anand, and A. Singh, "Artificial neural network-based deep learning model for COVID-19 patient detection using X-ray chest images," Journal of Healthcare Engineering, vol. 2021, Article ID 5513679, 16 pages, 2021.

[45] M. D. K. Hasan, S. Ahmed, Z. M. E. Abdullah et al., "Deep learning approaches for detecting pneumonia in COVID-19 patients by analyzing chest X-ray images," Mathematical Problems in Engineering, vol. 2021, Article ID 9929274, 8 pages, 2021.

[46] L. Gaur, U. Bhatia, N. Z. Jhanjhi, G. Muhammad, and M. Masud, "Medical image-based detection of COVID-19 using deep convolution neural networks," Multimedia Systems, vol. 11, pp. 1-10, 2021.

[47] R. M. Pereira, D. Bertolini, L. O. Teixeira, C. N. Silla, and Y. M. G. Costa, "COVID-19 identification in chest X-ray images on flat and hierarchical classification scenarios," Computer Methods and Programs in Biomedicine, vol. 194, Article ID 105532, 2020.

[48] H. Ma and J. Li, "A sub-linear time algorithm for approximating k-nearest-neighbor with full quality guarantee," Theoretical Computer Science, vol. 857, pp. 59-70, 2021.

[49] Y. Ruan, Y. Xiao, Z. Hao, and B. Liu, "A nearest-neighbor search model for distance metric learning," Information Sciences, vol. 552, pp. 261-277, 2021.

[50] R. Ghawi and J. Pfeffer, "Efficient hyperparameter tuning with grid search for text categorization using $\mathrm{kNN}$ approach with BM25 similarity," Open Computer Science, vol. 9, no. 1, pp. 160-180, 2019.

[51] Y. Li, X. Wang, and J. Wei, "Pruning stack algorithm and complexity analysis," in Proceedings of the 2007 International Symposium on Signals, Systems and Electronics, pp. 463-466, Montreal, QC, Canada, July 2007.

[52] M. Sandler, A. Howard, M. Zhu, A. Zhmoginov, and L.-C. Chen, "MobileNetV2: inverted residuals and linear bottlenecks," http://arxiv.org/abs/1801.04381.

[53] H. Dalianis, "Evaluation metrics and evaluation," in Clinical Text Mining, pp. 45-53, Springer International Publishing, Cham, 2018. 